\title{
Optimisation of a Small Non Controlled Wind Energy Conversion System for Stand-Alone Applications
}

\author{
Miguel Lopez, Philippe Dessante, Dario Morales, Jean-Claude Vannier and Daniel Sadarnac \\ Département Electrotechnique et Systèmes d'Energie \\ École Supérieure d'Electricité \\ 91190 Gif-sur-Yvette (France) \\ Phone number +33 (0) 169851 506, e-mail: miguel.lopez@supelec.fr, jean-claude.vannier@supelec.fr
}

\begin{abstract}
This article proposes a method to optimize the design of a small fixed-voltage wind energy conversion system (WECS). The system is composed of commercially available elements which are: a small horizontal axe wind turbine, a onestage gearbox, a permanent magnet synchronous generator, a diode bridge and a battery bank. As there are no controlled devices on the system, the design must be carefully done to find the configuration that maximizes both, the system utilization and the system's output power. Using the mechanical and the electrical power equations, an optimization problem is proposed. This problem is aimed to find the optimal combination of the gearbox ratio and the battery voltage in order to extract the maximum amount of energy form the WECS. The mechanical power is modelled using a new proposed power coefficient function approximation. The constrained optimization problem is then solved by a MATHEMATICAC genetic algorithm based routine. Results are shown and discussed.
\end{abstract}

\section{Key words}

Wind energy conversion system, stand-alone application, nonlinear optimization.

\section{Nomenclature}

$P_{t} \quad$ Wind turbine mechanical power $(\mathrm{W})$

$A \quad$ Wind turbine blade sweeping surface $\left(\mathrm{m}^{2}\right)$

$R \quad$ Wind turbine blade radius (m)

$C_{p} \quad$ Wind turbine power coefficient (-)

$\lambda \quad$ Tip-speed ratio $(-)$

$\Omega \quad$ Wind turbine rotor angular speed (rpm)

$v \quad$ Wind speed $[\mathrm{m} / \mathrm{s}]$

$M \quad$ Gearbox transmission ratio

$P_{m} \quad$ Generator electrical power (W)

$e \quad$ Generator electromotive force (V)

$u_{s} \quad$ Generator output voltage $(\mathrm{V})$

$i_{s} \quad$ Generator output current (A)

$\Omega_{\mathrm{G}} \quad$ Generator shaft rotation speed (rpm)

$\omega \quad$ Generator electrical rotation speed $(\mathrm{rad} / \mathrm{s})$

$\Psi_{\mathrm{r}} \quad$ Generator magnets induced flux (Wb)

$p \quad$ Generator pair of poles (-)

$Z_{s} \quad$ Generator impedance $(\Omega)$

$R_{s} \quad$ Generator winding resistance $(\Omega)$

$L_{s} \quad$ Generator leakage inductance $(\mathrm{H})$

$G \quad C_{p}$ approximation function Gain coefficient (-) $\begin{array}{ll}\lambda_{0} & C_{p} \text { approximation function Maximal } \lambda(-) \\ a & C_{p} \text { approximation function Coefficient }(-)\end{array}$

\section{Introduction}

Wind energy conversion systems (WECS) are currently the most competitive of the non conventional renewable energy technologies and the one which has the biggest growing rate [1], [2]. There is no doubt about the fact that wind power is becoming one of the most important renewable sources of electrical power [1]. The amount of electricity produced around the world using both largescale wind farms and small wind energy conversion systems is constantly growing.

Isolated sites or locations where the grid is unavailable are one of the most common commercial applications for small stand-alone wind systems [3], [4].

It is well known that controlled variable-speed structures allow maximizing the amount of energy produced by the WECS [4], [5]. Nevertheless, these systems are more complex, expensive, they require additional electric power conversion stages and specially designed control schemes.

When dealing with energy conversion systems that are going to be installed in isolated sites, especially those sites where access is difficult, system's simplicity helps reducing maintenance cost and increasing system's reliability.

In this article, the design of a simple non-controlled WECS with minimum components is optimized. It is assumed that the designed system will be used in standalone applications. From the system's model, equations for the wind turbine's mechanical power and the generator's electrical power are obtained. These expressions are dependant on the different system parameters and variables. The electrical power delivered to the load depends on the steady-state rotational speed of the system. In the fixed-voltage WECS, the rotational speed at each wind speed is dependent on some design parameters such as the gearbox ratio and the battery voltage. Thus, the objective is to maximize the output 
power of the proposed system. The problem is solved by finding the optimal combination of the gearbox transmission ratio and battery bank voltage.

The system's steady state model is described in section II. In the third section, the optimisation problem is explained and the proposed solution method is exposed. Results are summarized and shown in section IV.

\section{System Model}

The system under study is shown in Fig. 1. It is composed of a three-blade horizontal-axe wind turbine that takes the energy from a mass of moving air, a speedup gearbox to match the wind turbine (WT) and the generator rotor speeds, a permanent magnet synchronous machine (PMSM) for mechanical to electrical conversion, a diode bridge for AC to DC conversion and a battery bank for energy back-up and storage. It is supposed that the load consumes all the generated energy.

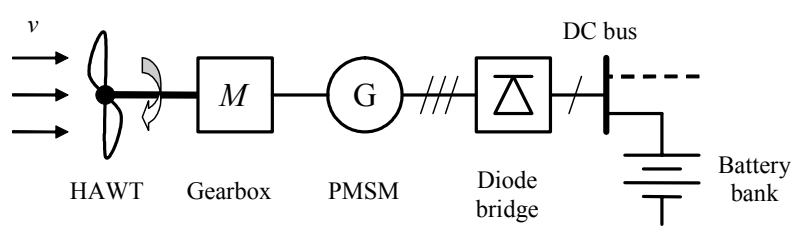

Fig. 1. Wind energy conversion system for stand-alone application with energy storage.

\section{A. Mechanical system}

The mechanical power $P_{t}$ that a wind turbine can extract from a mass of moving air that flows through it is given by:

$$
P_{t}=\frac{1}{2} \rho \cdot A \cdot C_{p}(\lambda) \cdot v^{3}
$$

$\rho$ is the air density, $A$ is the swept area of the wind turbine (WT) rotor, $v$ is the air speed (wind speed) and $C_{p}$ is the WT's power coefficient which depends on the tip speed ratio $(\lambda=\Omega R / v)$ [2], [3] and depends on the WT characteristics (horizontal/vertical axe, number and shape of blades, etc.).

The nonlinear characteristic $C_{p}(\lambda)$ can be approximated either by a polynomial function [5] or by a rational function [6]. The proposed form in equation (2) has the advantage of showing explicitly some information like the maximal tip-speed ratio for positive power coefficient $\lambda_{0}$ and the approximate value of the optimal tip-speed ratio for maximal power coefficient $C_{p}, \lambda^{*} \approx\left(\lambda_{0}-a\right)$. A simple least squares regression method is used to adjust the function coefficients $G$ and $a$.

$$
C_{p}(\lambda) \approx \frac{G \cdot \lambda\left(\lambda_{0}-\lambda\right)}{a^{2}+\left(\lambda_{0}-\lambda\right)^{2}}
$$

To adapt the slow rotating speed of the WT to the generator's high rotor speed, a gearbox must be used. For simplicity reasons, equation (3) is used to model the mechanical transmission system. $M$ represents the gearbox transmission ratio, $\Omega$ is the WT low-speed shaft and $\Omega_{G}$ is the electric machine faster shaft speed.

$$
\Omega_{G}=M \cdot \Omega
$$

The rotational speed of the generator shaft $\Omega_{G}$ and the electric rotational speed $\omega$ (here after the generator frequency) are related by the machine's pair of poles number $p\left(\omega=p \cdot \Omega_{G}\right)$. The WT mechanical power is expressed in terms of the transmission ratio $M$, the electrical frequency $\omega$ and the wind speed $v$ :

$$
P_{t}=\frac{1}{2} \frac{\rho A R G \omega\left(\lambda_{0} p M v-R \omega\right)}{(a p M v)^{2}+\left(\lambda_{0} p M v-R \omega\right)^{2}} \cdot v^{3}
$$

\section{B. Electrical system}

The generator is a three-phase permanent magnet synchronous machine that is modelled by a simple voltage source with series impedance. The equivalent circuit and the phasor diagram are shown in Fig. 2 [7]. The fundamental components of the voltage $u_{s}$ and the current $i_{s}$ are supposed to be in phase because the generator AC load is a simple silicon-diode bridge rectifier [8]. The machine voltage amplitude at fundamental frequency $\hat{u}_{s}$ is proportional to the battery bank voltage $U_{D C}$. The system batteries are lead-acid type because they are available even in extremely isolated places and they have easy and cheaper replacement than other batteries technologies.

From this model, a quadratic form for the generator's stator current equation is obtained [9], [10]. The stator current amplitude at fundamental frequency $\hat{\imath}_{S}$ can be calculated as follows:

$$
\hat{i}_{S}=\frac{-R_{S} \cdot \hat{u}_{S}+\sqrt{R_{S}^{2} \cdot \hat{u}_{S}^{2}+Z_{S}^{2} \cdot\left(e^{2}-\hat{u}_{S}^{2}\right)}}{Z_{S}^{2}}
$$

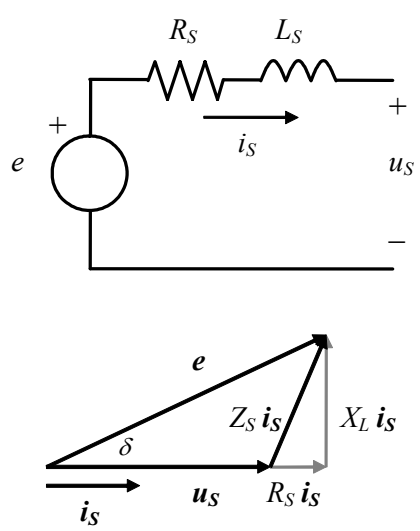

Fig. 2. PMSM equivalent circuit (generator reference direction) and associated phasor diagram. 
The amplitude of the electromotive force (EMF) $e$ is, in sinusoidal steady state, dependant on the flux induced by the magnets $\Psi_{r}$ and the frequency (6). The permanent magnets behave as a constant field excitation [7].

$$
e=\Psi_{r} \cdot \omega
$$

The generator impedance depends on the frequency as follows: $Z_{S}^{2}=R_{S}{ }^{2}+\left(\omega \cdot L_{S}\right)^{2}$. Replacing this in (5), the machine's electrical power can be expressed in terms of the frequency $\omega$ and the voltage amplitude $\hat{u}_{S}$ :

$$
P_{m}=\frac{1.5 \cdot \hat{u}_{s}}{R_{s}^{2}+\omega^{2} L_{s}^{2}}\left[\omega \sqrt{R_{s}^{2} \Psi_{r}^{2}+L_{s}^{2} \cdot\left(\omega^{2} \Psi_{r}^{2}-\hat{u}_{s}^{2}\right)}-R_{s} \hat{u}_{s}\right]
$$

\section{Optimization Problem}

The equations of the system's steady-state power (4) and (7) are the analytical expressions that allow the formulation of the main objective. The system equilibrium point is found at the crossing point between these two equations at the operating frequency (or wind speed). Assuming that the system losses are negligible, the output power is known by calculating the equilibrium points.

The coordinates of the crossing point depends on the value of the gearbox ratio $M$ and the battery voltage, which is proportional to the generator voltage $\hat{u}_{S}$. Thus, the system's output power is also dependant on these two variables.

The proposed approach consists of finding the value of $M$ and $\omega$ which maximizes the mechanical output power for a given wind speed and battery voltage. This is done knowing that both mechanical and electrical power must be equal.

The search of the solution is can't be done without some difficulties. It is necessary to use a special method because it can be found that:

1) Equation reduction is not possible. For both equations (4) and (7), it is not possible to extract the only independent variable that is common to both equations $\omega$. Thus, the variable replacement to reduce the system to only one equation cannot be done.

2) Parameterization with the only independent notcontrollable variable (v) is not realizable. There are infinite pairs $(M, \omega)$ that maximize $P_{t}$ for each wind speed value. It can be shown that only one wind turbine rotor speed value $\Omega^{*}$ does the maximization for a given $v$ value; but as $M$ and $\omega$ are both variable, the combination of both is represented by another equation (8).

$$
\Omega_{G}^{*}=M \cdot \Omega^{*}=M \frac{\omega}{p} \Rightarrow \omega^{*}=\left(p \cdot \Omega^{*}\right) \cdot M^{*}
$$

Therefore, the unique solution must be found by fixing (parameterize) other variable. Intrinsically, the proposed power structure cannot work in a permanent value for the rotor speed (frequency); so, the gearbox ratio or the battery voltage must be parameterized. As batteries are modular and can be easily associated for series and/or parallel electrical operation, it is the battery bank voltage $\hat{u}_{S}$ that is chosen for the parameterization. With this method, a set of mono-variable optimization problems are solved in terms of $\hat{u}_{S}$, for each selected wind speed.

\section{A. Constraints}

One-stage parallel gearbox systems have transmission ratios up to 1:5 [3] or 1:6 [2]. Equivalent planetary gearboxes have ratios up to $1: 12$, but they are more expensive; so, for small WT schemes, the parallel solution is regularly preferred. Higher transmission ratios are obtained adding further gear stages. There is also a minimum value for $M$, imposed by the turbine and the generator rotation speed rated values $\left(\Omega_{G e n, N} / \Omega_{N}\right)$. This way, the WT will not operate above its rated speed value even at generator's rated speed.

The generator rated values impose the upper limits of voltage, current and frequency. It is assumed that these restrictions are sufficient to keep the power generation lower or equal than the rated power. It is supposed that the WT can handle all the required mechanical power for low wind speeds $\left(v<v_{N}\right)$. Above that speed, the aerodynamic stall of the WT limits the mechanical power without further control. For maximal wind speed $\left(v_{\text {cut-off }}\right)$ the small WT furls automatically to protect itself of destructive high wind speeds.

The equation that models the generator's power (7) is valid only if the generator EMF is higher than a voltage threshold imposed by the battery bank voltage. This condition imposes a minimal rotational speed at which the PMSM begins to deliver power to the load. Thus, the battery bank voltage indirectly imposes the minimal wind speed (cut-in speed vcut-in).

The optimisation problem is defined as follows:

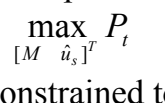

$$
\begin{aligned}
P_{t}(M, \omega, v) & =P_{m}\left(\hat{u}_{S}, \omega\right) \\
M & \in\left[\frac{\Omega_{\text {Gen, } N}}{\Omega_{N}}, 12\right] \\
\hat{u}_{S} & \in\left[0, \hat{u}_{N}\right] \\
\hat{\imath}_{S} & \in\left[0, \hat{i}_{N}\right] \\
\omega & \in\left[\frac{1}{\Psi_{r}} \hat{u}_{s}, \omega_{N}\right] \\
v & \in\left[\frac{R}{p \cdot \Psi_{r} \cdot \lambda_{0}} \cdot \frac{\hat{u}_{s}}{M}, v_{\text {cut-off }}\right]
\end{aligned}
$$

\section{Results}

It is important to point out that the independent variables in eq. (4) are the gearbox ratio $M$, the electric frequency $\omega$ and the wind speed $v$ while in (7) are the battery bank 
voltage $\hat{u}_{S}$ and the frequency $\omega$. Thus, the proposed procedure is the following:

1) Optimal mechanical power. For a given wind speed ( $v$ ) set, the optimal $P_{t}$ and $\Omega$ values can be found by a MATHEMATICAC geneticalgorithm based routine

2) Battery voltage parameterization. A set of battery group voltages $\hat{u}_{S}$ is chosen and used for each $v$ value.

3) Solving for $\omega$. With the optimal $P_{t}$ value, $P_{m}$ is solved to find the corresponding optimal frequency $\omega$ for each $\hat{u}_{S}$ value.

4) Gearbox ratio calculation. Using (8) and the $\Omega$ value, the gearbox ratio value $M$ is found.

The procedure points 3 and 4 are repeated for all the selected wind speed values.

In Table I the optimisation procedure results are summarized. Figure 3 shows the optimal power generation values and the corresponding rotor speed vs. the wind speed.

It can be noticed that for a wind speed of $9 \mathrm{~m} / \mathrm{s}$, the WT at optimal conditions delivers more than the generator's rated power. Superior wind speeds presents even higher maximal power values, so the analysis for optimal power is stopped.

The optimal relation between the WT rotor speed and the system output power is shown in fig 4 . This is easily obtained by using the optimal $\lambda$ value for each $v$ value.

TABLE I. Wind turbine mechanical power optimisation for selected wind speed values

\begin{tabular}{|c|c|c|}
\hline$v[\mathrm{~m} / \mathrm{s}]$ & $\Omega[\mathrm{rad} / \mathrm{s}]$ & $P_{t}[\mathrm{~W}]$ \\
\hline 3 & 11,3 & 67,0 \\
4 & 15,1 & 158,8 \\
5 & 18,9 & 310,1 \\
6 & 22,6 & 535,8 \\
7 & 26,4 & 850,8 \\
8 & 30,2 & 1270 \\
9 & 34,0 & 1808 \\
\hline
\end{tabular}

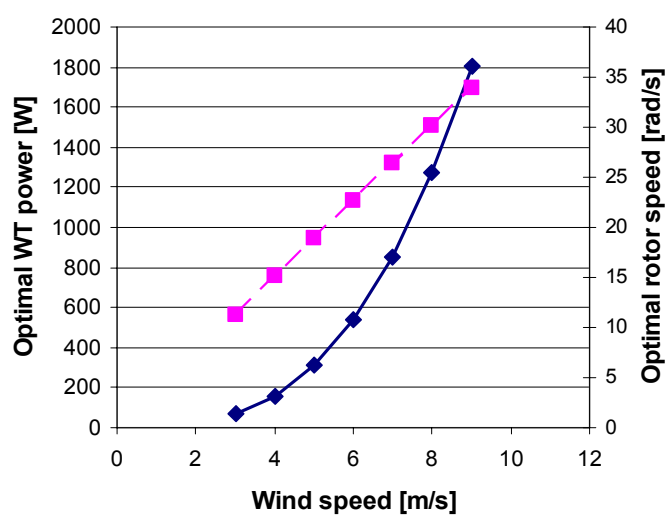

Fig. 3. WECS maximal power (solid line) and corresponding optimal rotor speed (dashed line) vs. wind speed

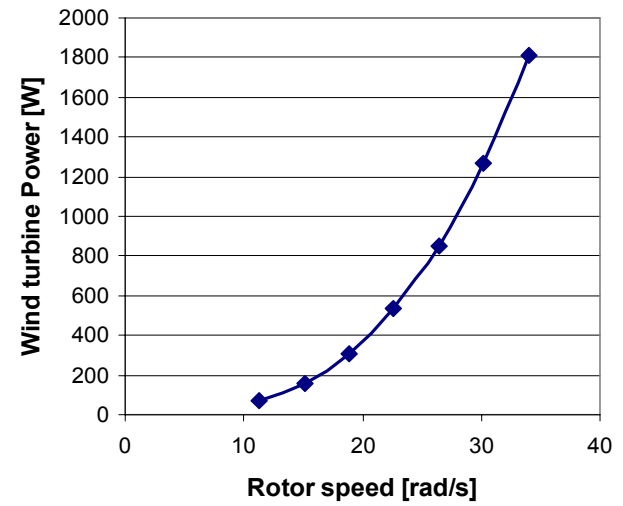

Fig. 4. Optimal rotor speed and power for the proposed wind generation system

The next step consists in obtaining the optimal values of the frequency (electric angular speed) by solving $P_{m}$ equal to $P_{t}$ for selected battery voltage values. These values are summarized in fig 5 . The optimal gearbox ratio is found using (8); figure 6 shows the corresponding optimal $M$ values.

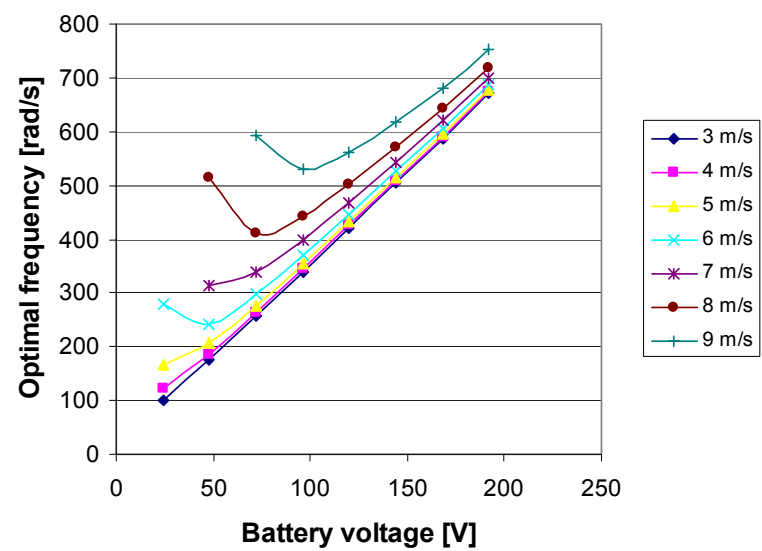

Fig. 5. Generator's optimal electric frequency vs. battery bank voltage, for the selected wind speed values.

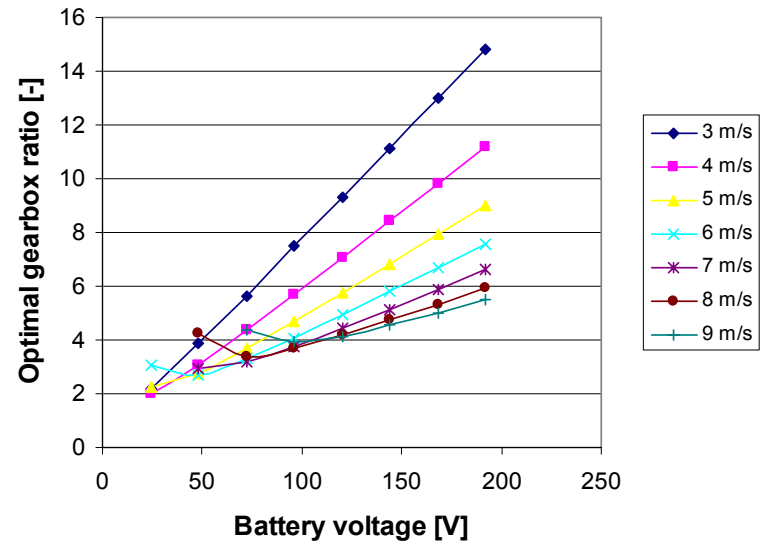

Fig. 6. Optimal gearbox transmission ratio obtained for the optimal frequencies and rotor speed values.

From fig. 5 it can be observed that, for low winds, the optimal frequency increases almost linearly with the battery voltage. The curve has a decreasing behaviour for 
wind speeds between 6 and $9 \mathrm{~m} / \mathrm{s}$ at low battery voltages. This is explained by the nonlinear evolution of the electric power with the battery voltage. In higher voltages, the increasing linear characteristic is found again.

The optimal $M$ curves in fig 6 are obtained directly from fig. 5 frequency values and equation (8); therefore, a similar behaviour is found: an almost linear behaviour with battery bank voltage is obtained for low winds and for high battery voltage for higher winds. But for the low battery voltages in the 6 to $9 \mathrm{~m} / \mathrm{s}$ wind range the decreasing characteristics is also found.

Furthermore, for each $v$ value, it can be shown that almost all $\hat{u}_{S}$ values have the same optimal power production. This is correct if we assume that we have the possibility of finding the right $M$ value that makes the system rotate at the optimal speed. Only a few of these $M$ values are discarded (higher than 12) when their value is too high and cannot be obtained without expensive multistage gearboxes.

Automatic gearboxes with multiple transmission ratios are not cost effective for the proposed low power generation system. Battery voltage variation implies the use of switches or another conversion stage that also increases the system cost. Therefore, it is necessary to use further criterion to choose a single $\left(M, \hat{u}_{S}\right)$ pair. One method is to maximize the WECS produced energy in the installation site. However, this can be done only if the wind conditions of the site, like the wind probability distribution function, are known. If this information or the site where the wind generation system will be placed are unknown, other selection method is to minimize the distance between the ideal $P(\Omega)$ curve (Fig. 4) and the generator power curve $P_{m}\left(\hat{u}_{S}, M, \omega\right)$. A minimum meansquare error method is suitable to fulfil this last criterion.

\section{Conclusion}

A method to optimize the design of an isolated small wind energy conversion system has been presented and studied. The objective to maximize the total energy produced by a simple wind energy conversion system without controlled components has been achieved. The method is based on the model of the wind turbine's mechanical power and the model of the electrical power produced by the PM generator. The mechanical power equation was obtained by modelling the wind turbine power coefficient with a simple rational function. Results were shown and discussed and a proposal was set to fix the battery voltage level and the gearbox ratio.

\section{Acknowledgement}

The authors would like to thank the Chilean Government and the Ecole Supérieure d'Electricité (Supelec) for their economical support in this research. Mr. Miguel Lopez is a "Presidente de la Republica de Chile" scholarship holder for his doctoral studies.

\section{References}

[1] World Energy Council, 2004 Survey of Energy Resources, Elsevier (2004).

[2] S. Mathew, Wind Energy: Fundamentals, Resource Analysis and Economics, Springer (2006).

[3] E. Hau, Wind Turbines: Fundamentals, Technologies, Application, Economics, Springer, (2006).

[4] A.M. De Broe, and S. Drouillet and V. Gevorgian, "A Peak Power Tracker for Small Wind Turbines in Battery Charging Applications", IEEE Trans. on Energy Conversion, Vol. 14, No. 4, pp 1630-1635, 1999.

[5] B.S. Borowy and Z.M. Salameh, "Dynamic Response of a Stand-Alone Wind Conversion System with Battery Energy Storage to a Wind Gust", IEEE Trans. on Energy Conversion, Vol. 12, No. 1, pp 73-78, March 1997.

[6] G.N. Kariniotakis and G.S. Stravrakakis, "A General Simulation Algorithm for the Accurate Assessment of Isolated Diesel - Wind Turbines Systems Interaction", IEEE Trans. on Energy Conversion, Vol. 10, No. 3, pp 584-590, September 1995.

[7] A. E. Fitzgerald, C. Kingsley Jr. And S. D. Umans, Electric Machinery, McGrawHill (1999).

[8] M. Rashid, Power Electronics Circuits, Devices and Applications, Pearson Prentice Hall (2004).

[9] D. Morales, "Optimalité des éléments d'un système décentralisé de production d'énergie électrique", $\mathrm{PhD}$ Thesis, Université de Paris XI, (2006).

[10] D. Morales, M. Lopez, and J.-C.Vannier, "Optimal Matching Between a Permanent Magnet Synchronous Machine and a Wind Turbine - Statistical Approach", in Proc. ICEM 2006.

\section{Appendix}

The wind turbine mechanical characteristics, the proposed power coefficient approximation parameters and the PMSM nominal values and characteristic parameters are listed below:

$\begin{array}{ll}\text { Wind turbine parameters } & \\ \text { Radius }(R) & =1.8 \mathrm{~m}(3.6 \mathrm{~m} \text { diameter }) \\ \text { Area }(A) & =10.18 \mathrm{~m}^{2} \\ \text { Max power coef. }\left(C_{p \text { Max }}\right) & =0.42 \\ \text { Optimal tip-speed ratio }\left(\lambda^{*}\right) & =6.8 \\ \text { Nominal wind speed }\left(v_{N}\right) & =12 \mathrm{~m} / \mathrm{s} \\ \text { Nominal rotation speed }\left(\Omega_{N}\right) & =700 \mathrm{rpm}\end{array}$

\section{Cp approximation function}

Gain $(G)$

factor $(a)$

Maximal tip-speed ratio $\left(\lambda_{0}\right)$

$$
\begin{aligned}
& =0.19 \\
& =1.56 \\
& =8.08
\end{aligned}
$$

\section{Electric generator}

Nominal torque $\left(T_{N}\right)$

Nominal rotation speed $\left(\Omega_{N}\right)$

Nominal power $\left(P_{N}\right)$

Nominal voltage $\left(v_{N}\right)$

Leakage resistance $\left(R_{S}\right)$

Leakage inductance $\left(L_{S}\right)$

Flux induced by magnets $\left(\Psi_{r}\right)$

Pairs of poles number $(p)$ 